\title{
Transdifferentiation of Neural Stem Cells, or Not?
}

\author{
A review of: Ying QL, Nichols J, Evans EP, Smith AG 2002 Changing potency by spontaneous fusion. Nature \\ 416(6880):545-548
}

$P_{c}^{\prime}$ LURIPOTENT STEM CELLS from the inner cell mass of the blastocyst can differentiate into cells of all three germ layers - the endoderm, mesoderm, and ectoderm. These layers are formed early in embryonic development and pools of more committed stem cells divide and further differentiate into organs and organized mature structures. Dividing progenitor cell populations have been isolated from most adult tissues. Originally, these cells were believed to be committed to differentiate solely into the cells of the tissue from which they were derived. More recently it has emerged that such tissuederived cells may be more plastic in their differentiation potential and be capable of generating a variety of cell types, and even producing cell types normally derived from a different germ layer - this is know as transdifferentiation. For example, neural stem cells are capable of generating hematopoietic progenitors (1), and bone marrow derived mesenchymal stem cells have been shown to differentiate into neural phenotypes (2), hepatocytes (3), and myocytes (4).

The concept of transdifferentiation has important implications and potentially allows the generation of tissuespecific progenitor cells from other tis-

\section{Nigel L. KenNEA \\ Huseyin Mehmet}

sues. Clearly, if adult derived cells could be induced to differentiate into any cell type, it would reduce the need for the use of ethically controversial embryonic stem cells for research and therapy. However, the concept of transdifferentiation is not universally accepted. Recently, two independent studies published in the same issue of Nature have provided an alternative explanation for transdifferentiation and raised doubts as to whether it really occurs $(5,6)$. Both used co-culture techniques with genetically labeled cells to enable cell tracking. This article discusses the paper of Ying et al. who took neural stem cells from transgenic mice and co-cultured them with modified embryonic stem (ES) cells. The aim of this series of experiments was to investigate whether ES cells could influence the de-differentiation of committed neural stem cells to a more pluripotent phenotype. In one experiment, Ying derived neural stem cells from transgenic mice expressing green fluorescent protein (GFP) and a gene conferring puromycin resistance, both under the control of the Oct-4 promoter. Oct-4 is a transcription factor expressed solely in pluripotent stem cells. These genetically labeled cells were co-cultured with modified ES cells expressing a fusion gene consisting of hygromycin phosphotransferase and herpes simplex thymidine kinase inserted into the Oct-4 locus. This gene when activated confers resistance to hygromycin, and sensitivity to ganciclovir.

Following co-culture, the original ES cells were selected against by adding puromycin to the growth medium, and after 2-4 weeks proliferating ESlike cells emerged that expressed the GFP transgene derived from the neural cell cultures. Since GFP expression was driven by Oct-4 activity, it seemed that de-differentiation of neural stem cells to ES cells had occurred. Indeed, the resulting cells from these experiments expressed ES markers, displayed similar culture requirements to ES cells and could be differentiated into a range of cell types including extra-embryonic endoderm, cardiac myocytes and neurons in vitro.

However, on further examination, the resulting cells were also resistant to hygromycin, and sensitive to ganciclovir. In other words the emerging population exhibited the sensitivity and 
resistance phenotypes of both the original cell types, suggesting that they were neural stem cell and ES cell hybrids. This possibility was further reinforced by the morphology of the resulting cells, which were different to native ES cells; specifically, they had larger nuclei and more abundant nucleoli. Chromosomal analysis confirmed the possibility of cell fusion. Karyotypic analysis of 18 independent cell isolates revealed tetraploidy, or near tetraploidy, in all cases.

It could be argued that cell fusion cannot explain the in vivo observations where large numbers of progenitors seem to undergo transdifferentiation. Paradoxically, this seems to be reinforced by the Ying study, where hybrid formation by cell fusion was a relatively rare event occurring at a maximum frequency of only 1 in 10,000 neural cells plated. However, this may reflect the stringent selection process in the culture conditions and, moreover, it should not be ignored that cell fusion did occur spontaneously in these experiments under normal culture conditions. One might envisage that the frequency rate of cell fusion might be even higher in vivo, especially if the transplant environment is favorable. Whether transdifferentiation is a true property of progenitor cells or a confusing artefact, the possibility of cell fusion must be examined in future research claiming to demonstrate stem cell plasticity. This will be especially important in the context of long-term culture, co-culture or transplantation experiments.

1. Bjornson CR, Rietze RL, Reynolds BA, Magli MC, Vescovi AL 1999 Turning brain into blood: a hematopoietic fate adopted by adult neural stem cells in vivo. Science 283(5401):534-537

2. Mezey E, Chandross KJ, Harta G, Maki RA, McKercher SR 2000. Turning blood into brain: cells bearing neuronal antigens generated in vivo from bone marrow. Science 290(5497):1779-1782

3. Lagasse E, Connors H, Al Dhalimy M, Reitsma M, Dohse M, Osborne L, Wang X, Finegold M, Weissman IL, Grompe M 2000. Purified hematopoietic stem cells can differentiate into hepatocytes in vivo. Nat Med 6(11):1229-1234

4. Ferrari G, Cusella-De Angelis G, Coletta M, Paolucci E, Stornaiuolo A, Cossu G, Mavilio,F 1998. Muscle regeneration by bone marrow-derived myogenic progenitors. Science 279(5356):1528-1530

5. Ying QL, Nichols J, Evans EP, Smith AG 2002. Changing potency by spontaneous fusion. Nature 416(6880):545-548

6. Terada N, Hamazaki T, Oka M, Hoki M, Mastalerz DM, Nakano Y, Meyer EM, Morel L, Petersen BE, Scott EW 2002. Bone marrow cells adopt the phenotype of other cells by spontaneous cell fusion. Nature 416(6880):542-545.

The Weston Laboratory

Institute of Reproductive and Developmental Biology

Division of Paediatrics, Obstetrics and Gynaecology

Imperial College of Science, Technology and Medicine

Hammersmith Hospital Campus

Du Cane Road

London W12 ONN UK

DOI: 10.1203/01.PDR.0000030706.04968.CF 